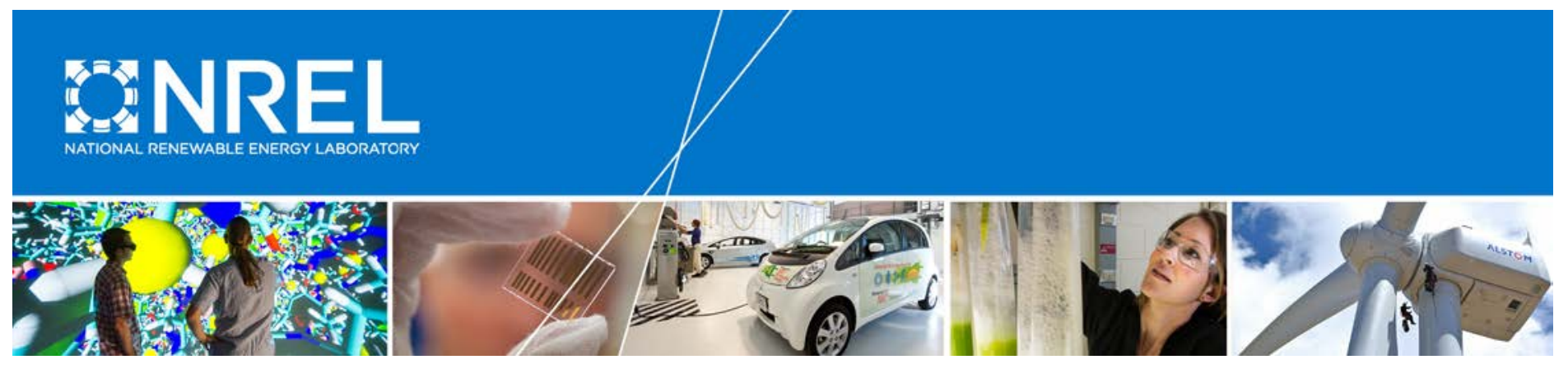

\title{
Optimizing Storage Operation for a Probabilistic Locational Marginal Pricing Forecast
}

\section{Preprint}

\author{
Aadil Latif, Dheepak Krishnamurthy, \\ and Bryan Palmintier \\ National Renewable Energy Laboratory
}

Presented at the 2018 IEEE International Conference on Probabilistic Methods Applied to Power Systems (PMAPS) Boise, Idaho

June 24-28, 2018

\section{Suggested Citation}

Latif, Aadil, Dheepak Krishnamurthy, and Bryan Palmintier. 2018. "Optimizing Storage Operation for a Probability Locational Marginal Pricing Forecast: Preprint." Golden, CO: National Renewable Energy Laboratory. NREL//CP5D00-70548. https://www.nrel.gov/docs/fy18osti/70548.pdf.

NREL is a national laboratory of the U.S. Department of Energy Office of Energy Efficiency \& Renewable Energy Operated by the Alliance for Sustainable Energy, LLC

This report is available at no cost from the National Renewable Energy Laboratory (NREL) at www.nrel.gov/publications.

\section{Conference Paper}

NREL/CP-5D00-70548

September 2018 


\section{NOTICE}

This work was authored by the National Renewable Energy Laboratory, operated by Alliance for Sustainable Energy, LLC, for the U.S. Department of Energy (DOE) under Contract No. DE-AC36-08GO28308. This work was supported by the Laboratory Directed Research and Development (LDRD) Program at NREL. The views expressed in the article do not necessarily represent the views of the DOE or the U.S. Government. The U.S. Government retains and the publisher, by accepting the article for publication, acknowledges that the U.S. Government retains a nonexclusive, paid-up, irrevocable, worldwide license to publish or reproduce the published form of this work, or allow others to do so, for U.S. Government purposes.

This report is available at no cost from the National Renewable Energy Laboratory (NREL) at www.nrel.gov/publications.

U.S. Department of Energy (DOE) reports produced after 1991 and a growing number of pre-1991 documents are available free via www.OSTI.gov. 


\section{Optimizing Storage Operation for a Probabilistic Locational Marginal Pricing Forecast}

\author{
Aadil Latif \\ National Renewable Energy Lab \\ aadil.latif@nrel.gov
}

\author{
Dheepak Krishnamurthy \\ National Renewable Energy Lab \\ dheepak.krishnamurthy@nrel.gov
}

\author{
Brian Palmintier \\ National Renewable Energy Lab \\ brian.palmintier@nrel.gov
}

\begin{abstract}
This paper explores the applicability of using dynamic programing (DP) and approximate dynamic programming (ADP) based methods for optimal dispatch of utility scale energy storage systems (ESS). In this study, the effectiveness of these approaches have been tested using the IEEE 13 node test feeder with distributed photovoltaics (PVs) and a utility scale storage system. In this work, a co-simulation based approach has been used to setup the experiment to be able to implement detailed ESS and network models. The results obtained from DP/ADP runs have been compared with three other control strategies both myopic and intelligent. Simulations results show that DP/ADP algorithms are a good candidate for optimal EES dispatch in terms of both solution quality and execution time.
\end{abstract}

Index Terms-energy storage, dynamic programming, approximate dynamic programming, co-simulation

\section{NOMENCLATURE}

$T \quad$ is the number of decision periods

$t \quad$ time index from 1 to $T$ and $t \in(1,24)$.

$x_{t} \quad$ is the state of system at time $t$.

$V_{\Pi}\left(x_{0}\right)$ is the expected value of policy $\Pi$ given the initial state $x_{0}$

$u_{t} \quad$ is the decision variable selected at time $t$.

$P^{\text {rated }}$ is the rated storage output.

$E^{\text {rated }}$ is the maximum energy the battery can store.

$w_{t} \quad$ represents the uncertainty pertaining to LMP forecast at time $t$.

$S_{t}^{b a t t}$ is the state of charge at the beginning of period $t$ and $\in(0,100)$.

$P_{t}^{b a t t}$ is the battery output (as percentage of rated power) at time $t$ and $\in(-100,100)$.

$L M P_{t}^{a}$ is the actual LMP signal at time $t$.

$L M P_{t}^{f}$ is the forecast LMP signal at time $t$.

$K^{b a t t}$ is the net profit for charing or discharging the battery at time period $t$.

$K^{r e v} \quad$ is the cost pertaining to penalty imposed on reverse power flow by the TSO.

$t_{c h r} \quad$ time trigger for ESS charging.

$t_{d c h r}$ time trigger for ESS discharging.

$L M P_{c h r}$ Price trigger for ESS charging.

$L M P_{d c h}$ Price trigger for ESS discharging.
$N \quad$ Population size for the PSO algorithm.

$G \quad$ Maximum number of iterations for the PSO algorithm.

$\iota \quad$ inertia value for the PSO algorithm.

$\phi \quad$ Correction factor for the PSO algorithm.

$\vec{P}_{\text {batt }_{i}}^{\text {best }}$ is the personal best experience of a swarm particle

$\vec{P}_{\text {batt }}^{\text {best }}$ is the global best experience for the particle swarm

\section{INTRODUCTION}

Traditionally, energy storage systems have been designed to store bulk energy with pumped hydro leading the market with about 90 percent share word wide [1]. Renewable energy technologies like wind and solar are inherently intermittent. To ensure power system stability and overcome the challenges associated with intermittent technologies, utilities are increasingly using energy storage systems capable of reacting to these intermittencies quickly [2]. Battery technologies like lithium-ion (Li-ion) or redox flow have the capability to ramp up to rated power within seconds [3]. This makes them an attractive option to mitigate challenges associated with intermittent renewable energy technologies. In recent years, lithium-ion (Li-ion) batteries have dominated the market with a share of about 94 percent in on-grid applications [4]. Liion batteries although more efficient when compared to flow batteries have limited lifespan [5] [6]. This makes redox flow batteries a great candidate for utility scale energy storage solution. In the US utility scale grid connected batteries are expect to increase six fold by the year 2022 [7].

Currently, there are only a handful utility owned and operated large scale batteries in the US [8]. For this reason, utilities have yet to fully understand how to best balance the multiple values of storage.

In literature a number of methods have been proposed for large grid connected battery storage systems.In [9], Hart and Sarkissian proposed a two stage optimization process to optimize both energy capacity and power rating of the ESS using an intra-hourly time interval. They simulate a number of scenarios to explore the impact of load and renewable energy generation variation on the proposed method. In [10] Tang and Jain proposed a method for economic dispatch where each generator has a storage device connected. They use game 
theory to show that use of a storage device does not increase options for strategic play. Reference [11] proposes a method to limit risk when dispatching storage. It proposes a gradient based method for optimal control. A number of researchers have used heuristic methods for optimal storage dispatch. In [12] authors used particle swarm (PSO) for optimal dispatch of multiple resources including battery storage systems. Simulation results show emission costs can be reduced by optimal utilization of renewable energy resources. Similarly in [13] the authors proposed using binary PSO for optimal economic dispatch battery storage systems.

A number of authors have used dynamic programming (DP) and approximate dynamic programming (ADP) based methods for optimal dispatch of storage devices [14]. In [15] Dutta and Overbye have proposed a method for computing optimal set points for storage dispatch connected to a wind farm. The formulated objective aims to mitigate generator's deviations from a set schedule, while considering intermittance associated with wind farms. In their work, the authors have used a stochastic dynamic programing framework for a fixed scheduling horizon. Results show that higher generation commitment is possible if the quality of probabilistic profiles is improved. Nascimento and Powell [16] similarly have used ADP for hourly dispatch of utility scale storage. The model takes into consideration daily and seasonal variations. In [17], the authors have proposed a DP based method for optimal storage operation. The work uses DC load flow to linearize the power flow equations thus enabling a semi-analytical solution formulation to the problem.

Although in the past, a lot of work has been done on the topic of optimal storage dispatch, previous work does not compare state-of-the-art methods with myopic approaches that are commonly employed by electric utilities taking uncertainty in price signal into consideration. Both myopic strategies and the PSO implementation can not integrate locational marginal price (LMP) uncertainty into the formulated problem unlike DP/ADP implementations. To ensure fair comparison, for methods unable to account for uncertainty, multiple LMP forecast profiles have been generated using Monte Carlo sampling. Simulations have been carried out for each of these profiles and the results have then been compared to the results obtained from DP/ADP implementation. Finally, results from myopic approaches have been compared with intelligent methods PSO and DP/ADP.

The paper is structured as follows. Section II provides problem overview, problem formulation and overview of the co-simulation setup. Section III details the case study. Section IV presents the results and related discussion. Finally in Section V, conclusions are drawn based on the presented results.

\section{PROBLEM FORMULATION}

In this paper, DP and ADP based approaches have been employed to optimize the dispatch schedule of a flow battery owned and operated by the utility. The optimizations have been carried out for the intra-hour energy market for a probabilistic
LMP forecast. In this work the following assumptions have been made.

- The probabilistic profile for the day ahead is known at time $t=0$ and does not change during the day.

- The initial state-of-charge at the beginning of the simulation is zero $\left(S_{0}^{b a t t}=0\right)$

- The state-of-charge at the end of a day's simulation is also zero $\left(S_{T+1}^{b a t t}=0\right)$

- The transmission system operator penalizes the utility in case of reverse power flow.

- The actual LMP price $L M P_{t}^{a}$ is only known for time periods less than or equal to the current time $t$.

At every time period, the utility can decide to either keep the storage system idle or charge or discharge it. This has been referred as the decision node in Figure 2. After the decision has been made, the cost is calculated using the probabilistic LMP profile referred to as uncertainty node in Figure 2. The actual and forecast LMP profiles used in this work are shown in Figure 1.

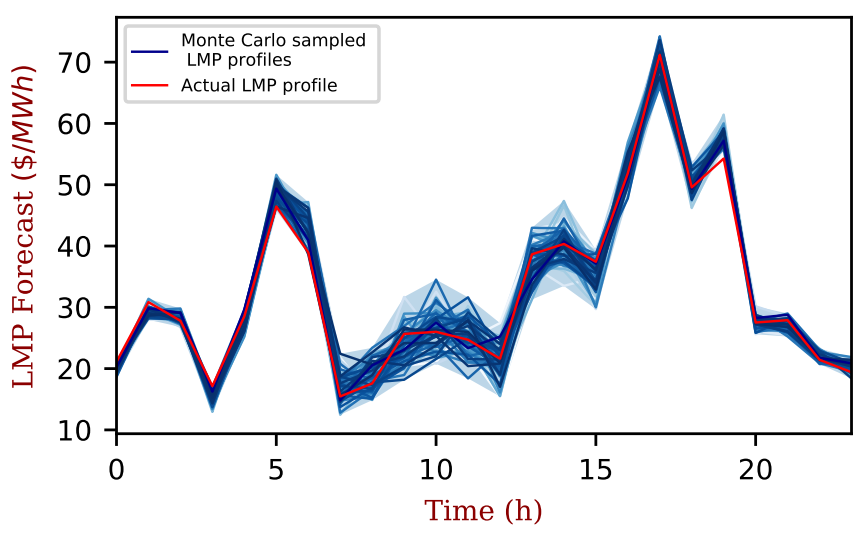

Fig. 1: Probabilistic (blue) LMP profiles generated using Monte Carlo sampling and the the actual LMP profile (red).

\section{A. Problem Structure}

Under the assumptions listed above, the problem described can be formulated as a dynamic program using Bellman's equation:

$$
\max _{\Pi} V_{\Pi}\left(x_{0}\right)=E\left\{\sum_{t=0}^{T-1} \gamma^{t} C_{t}\left(x_{t}, u_{t}, w_{t}\right)\right\}
$$

Where, the state of the system, $x_{t}$, is defined as:

$x_{t}=\left\{\begin{array}{lll}{\left[S_{t}^{\text {batt }},\right.} & P_{t}^{\text {batt }}, & L M P_{t}^{a}\end{array}\right]$ if $t_{\text {current }}=t$

The cost function for the problem has be defined as:

$C_{t}\left(x_{t}, u_{t}, w_{t}\right)=K^{b a t t}\left(x_{t}, u_{t}, w_{t}\right)-K^{r e v}\left(x_{t}, u_{t}, w_{t}\right)$ 


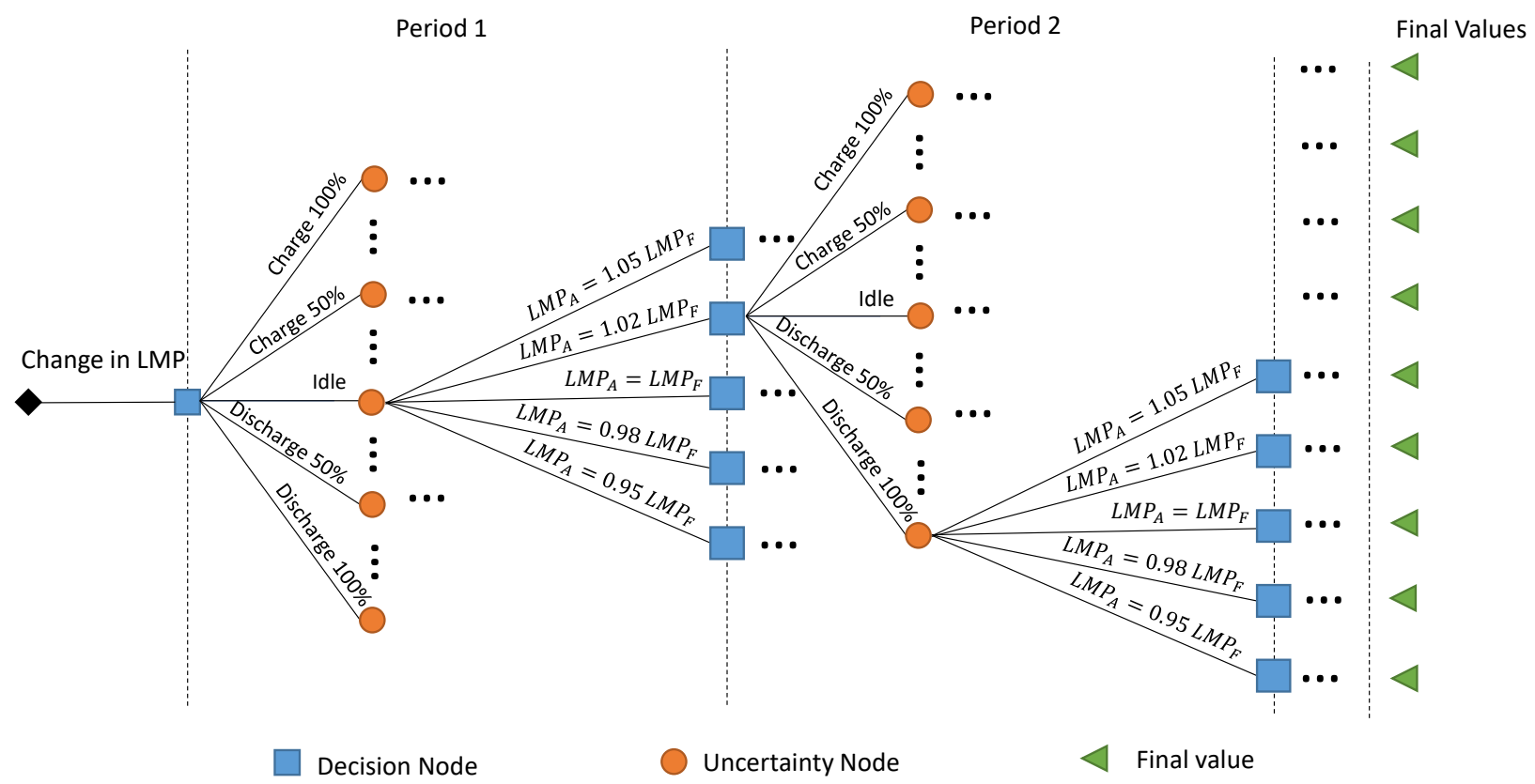

Fig. 2: Decision tree for the storage set point example

Where, the cost incurred due to charging, discharging or idling has been calculated using the equation:

$$
K^{\text {batt }}=L M P_{t}^{a c t} \cdot\left(\zeta^{d c h r} \cdot E_{t}^{d c h r}-\frac{E_{t}^{c h r}}{\zeta^{c h r}}\right)
$$

And, the cost incurred due to reverse power flow has been calculated using the equation:

$$
K^{r e v}=E_{t}^{r e v} \times R F P
$$

And the forecast LMP profile follows a normal distribution with:

$$
L M P_{t}^{f} \sim N\left(\mu_{t}, \sigma_{t}\right)
$$

\section{B. Simulation Setup}

We use OpenDSS [18] to simulate power flow on the grid and the behavior of power system devices, and Python-an open source high level scripting language-to implement the storage controller and facilitate communication between the optimization algorithms implemented in Matlab. OpenDSS is co-simulated into the system using the direct DLL interface with Python. Socket-based communication provides bidirectional communication among these tools and completes the co-simulation setup. This interface is used to update the system state and evaluate the cost of decisions for a given initial system state.

We compare multiple optimization algorithms, all implemented in Matlab. For DP/ADP, the dynamo open source implementation provides generic interfaces that treat the rest of the simulation setup as a black box, facilitating using a single problem formulation for both DP and ADP. We also implemented our own myopic decision policy and partical swarm optimization (PSO) algorithm for comparison with the
DP/ADP algorithms. Table II presents the parameters used for PSO.

\section{CASE STUDY}

For validation purposes we have used a modified version of the 13 node test feeder [19]. The modifications to the original test feeder have been detailed in [20]. Parameters for the battery model have been presented in Table I. Both myopic strategies and the PSO implementation can not integrate LMP uncertainty into the formulated problem unlike DP/ADP implementations. To ensure fair comparison, for methods unable to account for uncertainty, multiple LMP forecast profiles have been generated using Monte Carlo sampling. Simulations have been carried out for each of these profiles, and the results have then been compared to the results obtained from DP/ADP implementation. Finally, results from myopic approaches have been compared with intelligent methods PSO and DP/ADP.

\section{A. (Approximate) Dynamic programming (DP/ADP)}

The DP and ADP implementations make use of the structure of the problem by breaking a larger problem into smaller

TABLE I: Storage parameters

\begin{tabular}{lll}
\hline \multicolumn{1}{c}{ Parameter } & Value & Unit \\
\hline Rated power - $P^{\text {rated }}$ & 500 & $\mathrm{~kW}$ \\
Rated capacity - $S^{\text {rated }}$ & 2000 & $\mathrm{kWh}$ \\
Charging efficiency - $\zeta^{\text {chr }}$ & 80 & $\%$ \\
Discharging efficiency - $\zeta^{\text {dchr }}$ & 80 & $\%$ \\
Idling efficiency - $\xi^{\text {idle }}$ & 96 & $\%$ \\
Connected node & 671 & \\
\hline
\end{tabular}


TABLE II: Parameters used for PSO implementation

\begin{tabular}{lc}
\hline \multicolumn{1}{c}{ Parameter } & Value \\
\hline Population size $-N$ & {$[10,20,30]$} \\
Maximum iterations - $G$ & 20 \\
Inertia constant- $\iota$ & 0.1 \\
Correction constant $-\phi$ & 2 \\
\hline
\end{tabular}

TABLE III: Parameters used for the ADP-SBI algorithm

\begin{tabular}{lc}
\hline \multicolumn{1}{c}{ Parameter } & Value \\
\hline State sample / time period & [2-18], step 2 \\
Decision samples / State & 10 \\
Uncertainity sample / Decision & 5 \\
\hline
\end{tabular}

problems that can then be iteratively solved to converge to an optimal solution. In this paper, to generate an optimal storage policy for a day's operation, the DP/ADP programs divide the problem into 24 periods, each one hour long. DP is computationally expensive due to the combinatorial curse of dimensionality. ADP is a wide class of approaches that aim to reduce this computational burden by intelligent sampling and function approximation of the decision space rather than iteration through the entire decision space. Here we use one of the simplest ADP algorithms: sampled backward induction (SBI). SBI follows the general approach of traditional DP, but uses Monte Carlo sampling to only consider a subset of states, decisions, and uncertainties at each timestep. Our DP and ADP implementations works for discrete problems, so, in this work $P_{t}^{\text {batt }}$ has been discretized with steps of $10 \%$. Similarly, $S_{t}^{\text {batt }}$ has been discretized with steps of $5 \%$. Table III presents the ADP parameters used in this study.

\section{B. Time triggered (TT)}

The time triggered study case is a myopic approach where the storage starts charging at rated power at time $t=t_{c h r}$, and starts discharging at rated power as time $t$ approaches $t_{d c h r}$. In this work, the values for $t_{c h r}$ and $t_{d c h r}$ have been set at 2 and 14 hours respectively.

\section{Cost threshold triggered (ThrT)}

Cost threshold triggered is another myopic approach that uses the simple idea of buy low, sell high. The battery charges at rated power if the mean of the forecast LMP signal falls below the charge threshold, $L M P_{c h r}$ and discharges if the mean of the forecast LMP signal crosses below the discharge threshold $L M P_{d c h r}$. If the price signal is between the two thresholds, the storage maintains in an idling state. In this work, the values for $L M P_{c h r}$ and $L M P_{d c h r}$ have been set at $30 \$ / M W h$ and $40 \$ / M W h$ respectively.

\section{Particle swarm optimization (PSO)}

The PSO implementation aims to use swarm intelligence to converge to an optimal solution. PSO algorithm has also been implemented in MATLAB and uses the same co-simulation
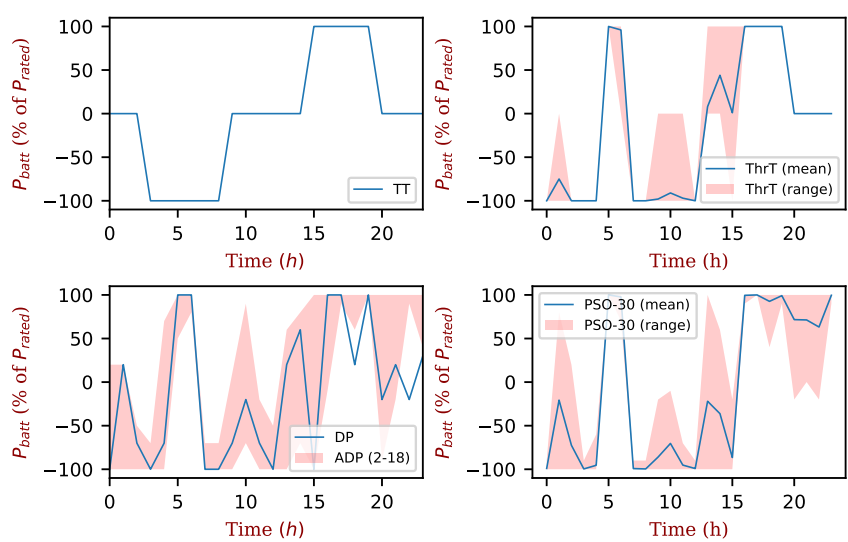

Fig. 3: Storage charge / discharge policy generated using different control strategies. The shaded area shows variability in generated policy for Monte Carlo scenarios for Thrt and $P S O-30$ scenarios. For $D P / A D P$ plot shaded area shows variability associated with varying state samples per iteration.

to communicate with OpenDSS. The pseudo code for the implementation has been presented in Algorithm 1. It is important to note that the PSO implementation also optimizes the discrete problem defined for DP and ADP methods. The cost function however has been modified to add a charge state penalty term.

$$
C_{t}^{P S O}\left(x_{t}, u_{t}, w_{t}\right)=-C_{t}\left(x_{t}, u_{t}, w_{t}\right)+\left(S_{T+1}^{b a t t}\right)^{2}
$$

This modification ensures that the SOC at the end of the day is zero. This enforces this key assumption and makes the PSO optimization problem equivalent to the DP/ADP problem, thus facilitating direct comparisons.

\section{RESULTS AND DisCUSSION}

Figure 3 shows the charge / discharge policy for the four test cases. The first subplot is for time triggered charge and

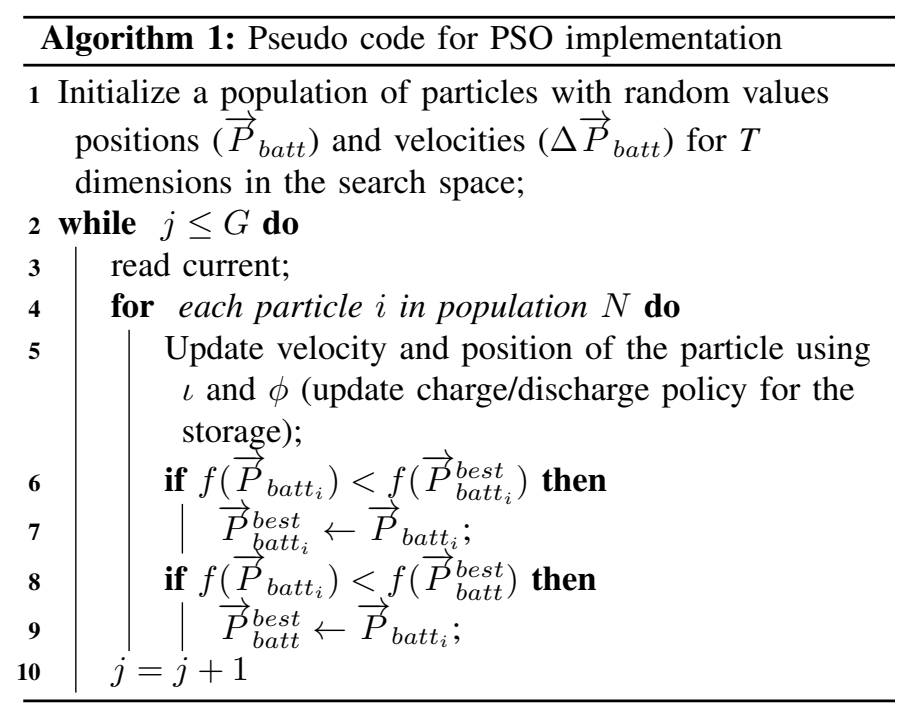


discharge. This myopic approach aims to utilize the evening peak to offset the costs pertaining to charging and system losses. However, this strategy has no insight of the price signal. This may lead to inefficient usage of the storage system as is evident from Figure 4.

The second subplot in Figure 3 is for the charge / discharge policy for the cost-triggered strategy. This control strategy although not intelligent, does use the LMP forecast. As a result it is able to improve upon the performance of time trigger myopic strategy (see Figure 4). This strategy makes use of the expected morning and evening price peaks, selling when the expected price is high and buying when low.

The lower left subplot in Figure 3 is the optimal policy generated by the DP algorithm. DP, unlike the first two methods, is an intelligent method thats takes into consideration the probability distribution of the LMP forecast. This added insight enables the DP algorithm to outperform the two myopic strategies. The average expected profit is $140 \%$ and $20 \%$ higher than $T T$ and $T h r T$ strategies respectively. Figure 5 compares the performance of the ADP algorithm with the DP algorithm. Instead of exploring the entire state, decision and uncertainty space, ADP algorithm randomly samples these spaces for a predefined number of times. In this work, the number of samples per state is varied while keeping the number of decision and uncertainty samples constant. For a small number of state samples the expected profit is much higher when compared to the DP solution (Figure 5). This is an artifact of the overly optimistic estimation resulting from incomplete exploration of the state space. These algorithmic guesses showing higher profits would not be realized if attempted with a more realistic scenario space; however, this discrepancy does provide a clear visualization of ADP convergence. As the number of state samples increase, the expected profit value converges to the value calculated using the DP method. This increased accuracy however, comes at the cost of increased execution time.

The lower right subplot in Figure 3 presents optimal policies generated using PSO for the generated Monte Carlo scenarios.

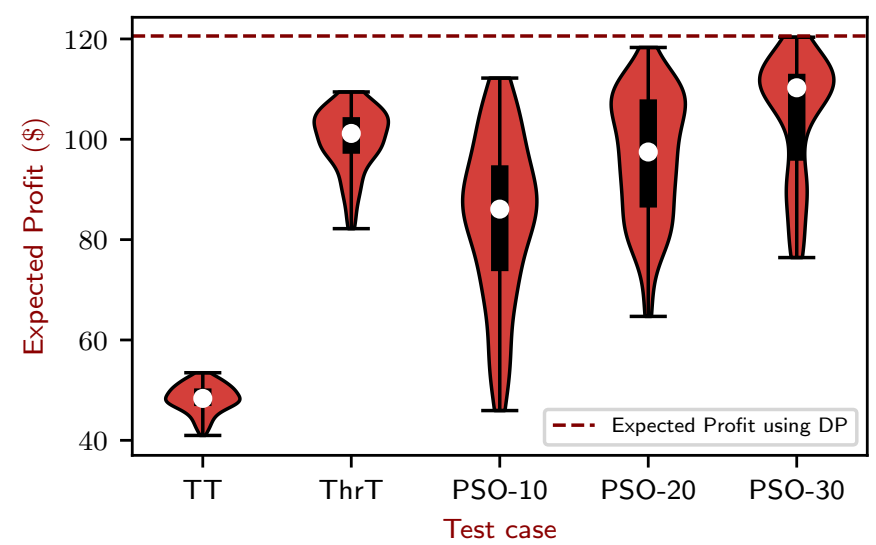

Fig. 4: A comparison of the expected profit calculated for all Monte Carlo scenarios using myopic and intelligent methods

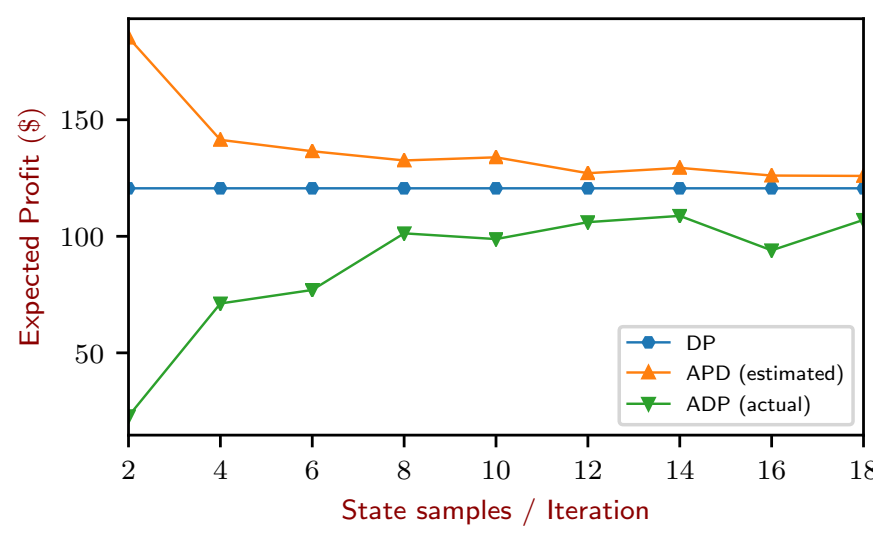

Fig. 5: Convergence plot for ADP as a function of the number of state samples. Here the overly optimistic expected profits with incomplete state explorations rapidly converge to more realistic values nearly matching DP with about 10 or more state samples per iteration.

Figure 6 shows PSO's convergence performance for three simulation runs with varying population size. Increasing population size does not ensure improved optimal solution as is the case for PSO-20 in Figure 6. The is because heuristic optimization methods like PSO may suffer from premature convergence. For a large number of simulation runs however, increasing population size does improve the performance of the algorithm (Figure 4). The average expected profits obtained from PSO are lower than the expected profit calculated using DP. One reason for poor convergence might be that PSO is not well suited for discrete problems.

Figure 7 shows the execution time for each test case. Results show that PSO scales poorly when the swarm size is increased. ADP on the other hand scales well as the number of state samples are increased. In these results, the relatively small problem size enables DP to take about the same computation time as ADP, however, the sampling-based ADP approaches are expected to scale better with larger and/or

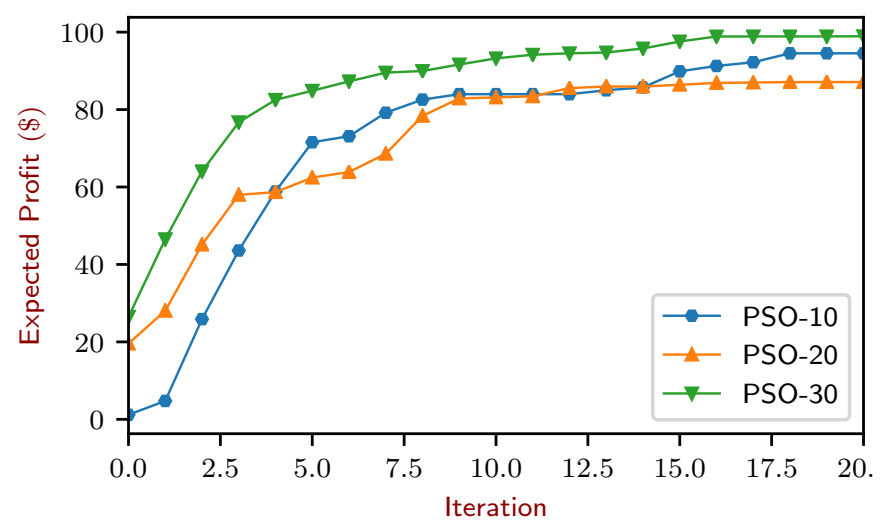

Fig. 6: Impact of increasing population size on PSO convergence 


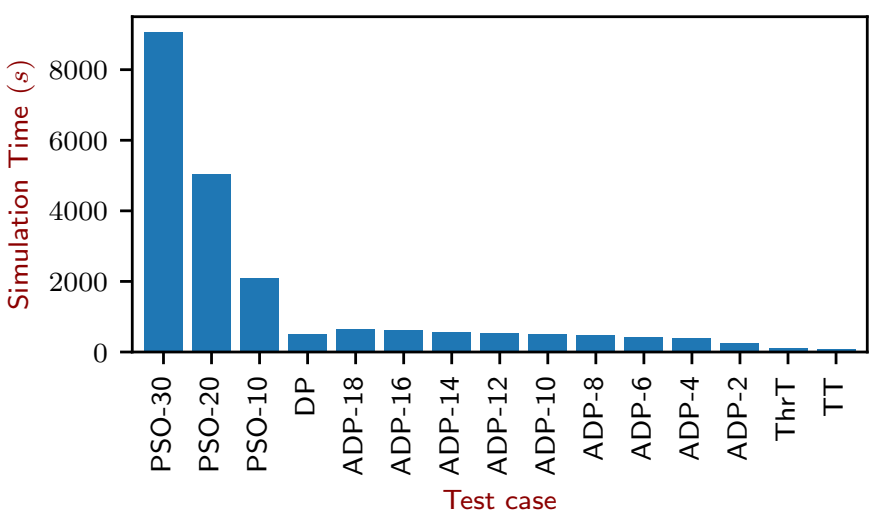

Fig. 7: Execution times for the implemented test cases.

more finely discretized problems where DP's combinatorial explosion increases simulation time exponentially. Across all algorithms, the run time is largely driven by the time required for OpenDSS simulations.

\section{CONCLUSIONS}

This paper explores the applicability of using dynamic programing (DP) and approximate dynamic programming (ADP) based methods for optimal dispatch of utility scale energy storage systems (ESS). In this study, the effectiveness of these approaches have been tested using the IEEE 13 node test feeder with distributed PVs and a utility scale storage system. In this work, a co-simulation based approach has been used to setup the experiment to be able to implement detailed ESS and network models. We compare these approaches to two simple myopic control strategies and various particle swarm optimization configurations. In these simulations, DP and ADP algorithms provide significantly increased expected profits $10-200 \%$ compared to the myopic approaches and 1$160 \%$ compared to PSO in significantly lesser computation time.

\section{ACKNOWLEDGMENT}

This work was supported by the U.S. Department of Energy under Contract No. DE-AC36-08-GO28308 with Alliance for Sustainable Energy, the Manager and Operator of the National Renewable Energy Laboratory (NREL) though the Laboratory Directed Research and Development Program. The U.S. Government retains and the publisher, by accepting the article for publication, acknowledges that the U.S. Government retains a nonexclusive, paid-up, irrevocable, worldwide license to publish or reproduce the published form of this work, or allow others to do so, for U.S. Government purposes.

\section{REFERENCES}

[1] IRENA, "Battery Storage for Renewables : Market Status and Technology Outlook," Tech. Rep. January, 2015. [Online]. Available: https://www.irena.org/documentdownloads/publications/irena_battery _storage_report_2015.pdf
[2] B. A. Antal, "Pumped Storage Hydropower: A Technical Review," Ph.D. dissertation, University of Colorado Boulder, 2014. [Online]. Available: http://www.ucdenver.edu/facultystaff/dmays/3414/Documents/Antal-MS-2014.pdf

[3] T. Shibata, T. Kumamoto, Y. Nagaoka, K. Kawase, and K. Yano, "Redox flow batteries for the stable supply of renewable energy," SEI Technical Review, vol. 76, p. 14, 2013.

[4] Greentech Media, "U . S . Energy Storage Monitor : Q2 2017 Executive Summary," no. June, 2017.

[5] M. Uhrig, S. Koenig, M. R. Suriyah, and T. Leibfried, "Lithium-based vs. vanadium redox flow batteries a comparison for home storage systems," Energy Procedia, vol. 99, no. Supplement C, pp. 35 - 43, 2016, 10th International Renewable Energy Storage Conference, IRES 2016, 15-17 March 2016, Dsseldorf, Germany. [Online]. Available: http://www.sciencedirect.com/science/article/pii/S1876610216310566

[6] B. Dunn, H. Kamath, and J.-M. Tarascon, "Electrical energy storage for the grid: A battery of choices," Science, vol. 334 , no. 6058, pp. 928-935, 2011. [Online]. Available: http://science.sciencemag.org/content/334/6058/928

[7] Energy Storage Systems, "Beyond Four Hours. The transition to a more flexible, and valuable, long-duration energy storage asset." Energy Storage Systems, Portland, Tech. Rep., 2016. [Online]. Available: http://www.essinc.com/wp-content/uploads/2016/11/BeyondFour-Hours_ESS-Inc-White-Paper_12_2016_mr.pdf

[8] D. Hart and A. Sarkissian, "Deployment of grid-scale batteries in the united states," Schar School of Policy and Government, George Mason University, Tech. Rep., 2016.

[9] K. Baker, G. Hug, and X. Li, "Optimal storage sizing using twostage stochastic optimization for intra-hourly dispatch," in 2014 North American Power Symposium (NAPS), Sept 2014, pp. 1-6.

[10] W. Tang and R. Jain, "Dynamic economic dispatch game: The value of storage," IEEE Transactions on Smart Grid, vol. 7, no. 5, pp. 23502358, Sept 2016.

[11] J. Qin, H. I. Su, and R. Rajagopal, "Storage in risk limiting dispatch: Control and approximation," in 2013 American Control Conference, June 2013, pp. 4202-4208.

[12] A. Karthikeyan, K. Manikandan, and P. Somasundaram, "Economic dispatch of microgrid with smart energy storage systems using particle swarm optimization," in Computation of Power, Energy Information and Commuincation (ICCPEIC), 2016 International Conference on. IEEE, 2016, pp. 783-789.

[13] E. D. Piedad Jr, M. E. F. Montilla, and M. J. E. Ortega, "Optimal scheduling of battery energy storage for grid-connected load using photovoltaic system (pv) via binary particle swarm optimization (bpso)," Recoletos Multidisciplinary Research Journal, vol. 4, no. 2, 2017.

[14] A. Papavasiliou, Y. Mou, L. Cambier, and D. Scieur, "Application of Stochastic Dual Dynamic Programming to the Real-Time Dispatch of Storage under Renewable Supply Uncertainty," IEEE Transactions on Sustainable Energy, pp. 1-1, 2017. [Online]. Available: http://ieeexplore.ieee.org/document/8024054/

[15] S. Dutta and T. J. Overbye, "Optimal storage scheduling for minimizing schedule deviations considering variability of generated wind power," in 2011 IEEE/PES Power Systems Conference and Exposition. IEEE, mar 2011, pp. 1-7. [Online]. Available: http://ieeexplore.iee.org/document/5772521/

[16] J. Nascimento and W. B. Powell, "An optimal approximate dynamic programming algorithm for concave, scalar storage problems with vectorvalued controls," IEEE Transactions on Automatic Control, vol. 58, no. 12, pp. 2995-3010, Dec 2013.

[17] J. Qin and R. Rajagopal, "Dynamic programming solution to distributed storage operation and design," IEEE Power and Energy Society General Meeting, pp. 1-5, 2013.

[18] R. C. Dugan and T. E. McDermott, "An open source platform for collaborating on smart grid research," in Power and Energy Society General Meeting, 2011 IEEE. IEEE, 2011, pp. 1-7.

[19] W. H. Kersting, "Radial distribution test feeders," in Power Engineering Society Winter Meeting, 2001. IEEE, vol. 2. IEEE, 2001, pp. 908-912.

[20] B. Palmintier, D. Krishnamurthy, and H. Wu, "Design flexibility for uncertain distributed generation from photovoltaics," in Innovative Smart Grid Technologies Conference (ISGT), 2016 IEEE Power \& Energy Society. IEEE, 2016, pp. 1-5. 